PROCEEDINGS OF THE

AMERICAN MATHEMATICAL SOCIETY

Volume 124, Number 10, October 1996

\title{
THE DISTANCE FROM THE APOSTOL SPECTRUM
}

\author{
V. KORDULA AND V. MÜLLER
}

(Communicated by Palle E. T. Jorgensen)

\begin{abstract}
If $T$ is an s-regular operator in a Banach space (i.e. $T$ has closed range and $\left.N(T) \subset R^{\infty}(T)\right)$ and $\gamma(T)$ is the Kato reduced minimum modulus, then$$
\lim _{n \rightarrow \infty} \gamma\left(T^{n}\right)^{1 / n}=\sup \{r: T-\lambda \text { is s-regular for }|\lambda|<r\} .
$$

Let $x$ be an element of a Banach algebra $A$. The spectral radius of $x$ is given by the well-known spectral radius formula: $r(x)=\lim _{n \rightarrow \infty}\left\|x^{n}\right\|^{1 / n}$.

There are a number of generalizations of this formula. If we set $d(x)=\inf \{\|x y\|$ : $y \in A,\|y\|=1\}$ and denote by $\tau_{l}(x)=\{\lambda \in \mathbf{C}: d(x-\lambda)=0\}$ the left approximate point spectrum of $x$, then $\operatorname{dist}\left\{0, \tau_{l}(x)\right\}=\lim _{n \rightarrow \infty} d\left(x^{n}\right)^{1 / n}$; see [13], [9]. In particular, in the algebra $B(X)$ of all bounded linear operators in a Banach space $X$ this gives formulas for radii of boundedness below or surjectivity:

$$
\sup \{r: T-\lambda \text { is bounded below for }|\lambda|<r\}=\lim _{n \rightarrow \infty} j\left(T^{n}\right)^{1 / n}
$$

and

$$
\sup \{r: T-\lambda \text { is onto for }|\lambda|<r\}=\lim _{n \rightarrow \infty} k\left(T^{n}\right)^{1 / n},
$$

where $j(T)$ and $k(T)$ are the moduli of injectivity and surjectivity of $T$ :

$$
j(T)=\inf \{\|T x\|: x \in X,\|x\|=1\} \quad \text { and } \quad k(T)=\sup \left\{r: T U_{X} \supset r U_{X}\right\},
$$

where $U_{X}$ is the closed unit ball in $X$.

For a bounded linear operator $T$ in a Banach space $X$ denote by $N(T)$ and $R(T)$ its kernel and range, respectively. Denote further $R^{\infty}(T)=\bigcap_{n=1}^{\infty} R\left(T^{n}\right)$ and $N^{\infty}(T)=\bigcup_{n=1}^{\infty} N\left(T^{n}\right)$.

The injectivity and surjectivity moduli of an operator which is bounded below (onto) are special cases of the Kato reduced minimum modulus [7]

$$
\gamma(T)=\inf \left\{\frac{\|T x\|}{\operatorname{dist}\{x, N(T)\}}: x \in X \backslash N(T)\right\}
$$

(for $T=0$ we define formally $\gamma(T)=\infty$ ).

The existence and the meaning of the $\operatorname{limit} \lim _{n \rightarrow \infty} \gamma\left(T^{n}\right)^{1 / n}$ in a more general setting were studied by Apostol [1] and Mbekhta [10].

Received by the editors October 14, 1994 and, in revised form, January 26, 1995.

1991 Mathematics Subject Classification. Primary 47A10, 47A53.

The research was supported by the grant No. 119106 of the Academy of Sciences of the Czech Republic. 
Definition. Let $T \in B(X)$. We say that $T$ is s-regular (= semi-regular) if $R(T)$ is closed and $N(T) \subset R^{\infty}(T)$.

The s-regular operators and closely related classes of operators were studied (under various names) by many authors; see $[3,4,5,6,8,16]$. We list some of the most important equivalent conditions for s-regular operators; see [11, 12].

Theorem. Let $T \in B(X)$ be an operator with a close range. The following conditions are equivalent:

(1) $T$ is s-regular,

(2) the function $\lambda \mapsto R(T-\lambda)$ is continuous at 0 in the gap topology,

(3) the function $\lambda \mapsto N(T-\lambda)$ is continuous at 0 in the gap topology,

(4) the function $\lambda \mapsto \gamma(T-\lambda)$ is continuous at 0 ,

(5) $\liminf _{\lambda \rightarrow 0} \gamma(T-\lambda)>0$,

(6) $N^{\infty}(T) \subset R(T)$,

(7) $N^{\infty}(T) \subset R^{\infty}(T)$.

Denote further $\sigma_{\gamma}(T)=\{\lambda \in \mathbf{C}: T-\lambda$ is not s-regular $\}$. The set $\sigma_{\gamma}(T)$ was studied by Apostol [1], Rakočevič [15], Mbekhta and Ouahab [11, 12] and Mbekhta [10]. The terminology is not unified; we suggest to call $\sigma_{\gamma}(T)$ the Apostol spectrum of $T$.

The Apostol spectrum $\sigma_{\gamma}(T)$ is always a non-empty compact subset of the complex plane, $\partial \sigma(T) \subset \sigma_{\gamma}(T) \subset \sigma(T)$ and $\sigma_{\gamma} f(T)=f \sigma_{\gamma}(T)$ for any function $f$ analytic in a neighbourhood of $\sigma(T)$.

If $T$ is an s-regular operator in a Hilbert space, then the $\operatorname{limit}_{\lim } \rightarrow \infty \gamma\left(T^{n}\right)^{1 / n}$ exists and

$$
\lim _{n \rightarrow \infty} \gamma\left(T^{n}\right)^{1 / n}=\operatorname{dist}\left\{0, \sigma_{\gamma}(T)\right\}=\sup \{r: T-\lambda \text { is s-regular for }|\lambda|<r\} ;
$$

see [1, Theorem 3.2] or [10, Theorem 3.1].

The aim of this paper is to prove equality (1) for operators in Banach spaces. This gives a positive answer to the conjecture of Rakočevič [15] and Mbekhta and generalizes the above-mentioned results for radii of injectivity and surjectivity.

Further, we study the essential version of this result.

If $T$ is a semi-Fredholm operator, then the $\operatorname{limit}_{\lim } \rightarrow \infty \gamma\left(T^{n}\right)^{1 / n}$ exists by [2] and it is equal to the semi-Fredholm radius of $T$ :

$$
\lim _{n \rightarrow \infty} \gamma\left(T^{n}\right)^{1 / n}=\sup \{r: T-\lambda \text { is semi-Fredholm for }|\lambda|<r\} ;
$$

see [17] and [2].

We prove a similar formula for essentially s-regular operators which generalizes the semi-Fredholm case.

The authors wish to thank M. Mbekhta for drawing their attention to the problem and for fruitful discussions concerning it.

Lemma 1. $T \in B(X)$ is s-regular if and only if there exists a closed subspace $M \subset X$ such that $T M=M$ and the operator $\tilde{T}: X / M \rightarrow X / M$ induced by $T$ is bounded below.

Proof. If $T$ is s-regular, then set $M=R^{\infty}(T)$. It is well known that $M$ is closed and (see e.g. [4, Theorem 3.4]) that $T M=M$ and $\tilde{T}: X / M \rightarrow X / M$ is bounded from below. 
Conversely, let $M$ be the subspace of $X$ with the required properties. Then $T M=M$ implies $M \subset R^{\infty}(T)$. If $T x=0$, then $\tilde{T}(x+M)=0$ and the injectivity of $\tilde{T}$ implies $x \in M$. Thus $N(T) \subset M \subset R^{\infty}(T)$.

It remains to prove that $T$ has closed range. Let $\pi: X \rightarrow X / M$ be the canonical projection. We show $R(T)=\pi^{-1} R(\tilde{T})$. If $y \in R(T), y=T x$ for some $x \in X$, then $\pi y=T x+M=\tilde{T}(x+M) \in R(\tilde{T})$ so that $R(T) \subset \pi^{-1} R(\tilde{T})$. If $y \in X$ and $\pi y \in R(\tilde{T})$, i.e. $y+M=T x+M$ for some $x \in X$, then $y \in R(T)$ since $M \subset R(T)$. Thus $R(T)=\pi^{-1} R(\tilde{T})$, which is closed since $R(\tilde{T})$ is closed and $\pi$ continuous.

Lemma 2. Let $T \in B(X)$, and let $M$ be a closed subspace of $X$ such that $T M=M$ and the operator $\tilde{T}: X / M \rightarrow X / M$ induced by $T$ is bounded below. Denote by $T_{1}: M \rightarrow M$ the restriction of $T$ to $M$. Then

$$
\lim _{n \rightarrow \infty} \gamma\left(T^{n}\right)^{1 / n}=\min \left\{\lim _{n \rightarrow \infty} \gamma\left(T_{1}^{n}\right)^{1 / n}, \lim _{n \rightarrow \infty} \gamma\left(\tilde{T}^{n}\right)^{1 / n}\right\} .
$$

Proof. The limits on the right-hand side exist by [17]. If $T^{n} x=0$, then $\tilde{T}^{n}(x+M)=$ 0, i.e. $x \in M$. Thus $N\left(T^{n}\right) \subset M$ and $N\left(T_{1}^{n}\right)=N\left(T^{n}\right)$. We have

$$
\begin{aligned}
\gamma\left(T_{1}^{n}\right) & =\inf \left\{\frac{\left\|T_{1}^{n} x\right\|}{\operatorname{dist}\left\{x, N\left(T_{1}^{n}\right)\right\}}: x \in M \backslash N\left(T_{1}^{n}\right)\right\} \\
& =\inf \left\{\frac{\left\|T^{n} x\right\|}{\operatorname{dist}\left\{x, N\left(T^{n}\right)\right\}}: x \in M \backslash N\left(T^{n}\right)\right\} \geq \gamma\left(T^{n}\right) .
\end{aligned}
$$

Further, since $T M=M$,

$$
\begin{aligned}
\gamma\left(\tilde{T}^{n}\right) & =\inf \left\{\frac{\left\|\tilde{T}^{n}(x+M)\right\|}{\|x+M\|}: x \notin M\right\}=\inf \left\{\frac{\left\|T^{n} x+M\right\|}{\operatorname{dist}\{x, M\}}: x \notin M\right\} \\
& \geq \inf \left\{\frac{\left\|T^{n} x\right\|}{\operatorname{dist}\{x, M\}}: x \notin M\right\} \geq \inf \left\{\frac{\left\|T^{n} x\right\|}{\operatorname{dist}\left\{x, N\left(T^{n}\right)\right\}}: x \notin M\right\} \geq \gamma\left(T^{n}\right) .
\end{aligned}
$$

Thus $\gamma\left(T^{n}\right) \leq \min \left\{\gamma\left(T_{1}^{n}\right), \gamma\left(\tilde{T}^{n}\right)\right\}$ and

$$
\limsup _{n \rightarrow \infty} \gamma\left(T^{n}\right)^{1 / n} \leq \min \left\{\lim _{n \rightarrow \infty} \gamma\left(T_{1}^{n}\right)^{1 / n}, \lim _{n \rightarrow \infty} \gamma\left(\tilde{T}^{n}\right)^{1 / n}\right\} .
$$

Denote

$$
s=\min \left\{\lim _{n \rightarrow \infty} \gamma\left(T_{1}^{n}\right)^{1 / n}, \lim _{n \rightarrow \infty} \gamma\left(\tilde{T}^{n}\right)^{1 / n}\right\}
$$

We prove $\liminf \operatorname{in}_{n \rightarrow \infty} \gamma\left(T^{n}\right)^{1 / n} \geq s$.

Let $n \geq 1, x=x_{0} \in R\left(T^{n}\right),\|x\|=1$, and let $s>\varepsilon>0$. Then $x+M \in R\left(\tilde{T}^{n}\right)$ and

$$
\left\|\tilde{T}^{-i}(x+M)\right\| \leq \gamma\left(\tilde{T}^{i}\right)^{-1}\|x+M\| \leq \gamma\left(\tilde{T}^{i}\right)^{-1} \quad(i=1, \ldots, n) .
$$

Thus there exist vectors $x_{i} \in \tilde{T}^{-i}(x+M)$ such that

$$
\left\|x_{i}\right\| \leq \gamma\left(\tilde{T}^{i}\right)^{-1}(1+\varepsilon) \quad(i=1, \ldots, n) .
$$

Denote $m_{i}=T x_{i+1}-x_{i}(i=0, \ldots, n-1)$. Then

$$
\left\|m_{i}\right\| \leq\|T\|\left\|x_{i+1}\right\|+\left\|x_{i}\right\| \leq(1+\varepsilon)\left[\|T\| \gamma\left(\tilde{T}^{i+1}\right)^{-1}+\gamma\left(\tilde{T}^{i}\right)^{-1}\right] \quad(i=0, \ldots, n-1) .
$$


Further, $\tilde{T}^{i}\left(m_{i}+M\right)=T^{i+1} x_{i+1}-T^{i} x_{i}+M=M$ so that $m_{i} \in M$ for each $i$. We have

$$
\begin{aligned}
\sum_{i=0}^{n-1} T^{i} m_{i} & =\left(T^{n} x_{n}-T^{n-1} x_{n-1}\right)+\left(T^{n-1} x_{n-1}-T^{n-2} x_{n-2}\right)+\cdots+\left(T x_{1}-x\right) \\
& =T^{n} x_{n}-x .
\end{aligned}
$$

Since $T_{1} M \rightarrow M$ is onto, there exist vectors $m_{i}^{\prime} \in M$ such that $T^{n-i} m_{i}^{\prime}=m_{i}$ and $\left\|m_{i}^{\prime}\right\| \leq(1+\varepsilon) \gamma\left(T_{1}^{n-i}\right)^{-1}\left\|m_{i}\right\|$. Thus

$$
T^{n}\left(x_{n}-\sum_{i=0}^{n-1} m_{i}^{\prime}\right)=T^{n} x_{n}-\sum_{i=0}^{n-1} T^{i} m_{i}=x
$$

and

$$
\left\|x_{n}-\sum_{i=0}^{n-1} m_{i}^{\prime}\right\| \leq(1+\varepsilon) \gamma\left(\tilde{T}^{n}\right)^{-1}+\sum_{i=0}^{n-1}(1+\varepsilon)^{2} \gamma\left(T_{1}^{n-i}\right)^{-1}\left[\|T\| \gamma\left(\tilde{T}^{i+1}\right)^{-1}+\gamma\left(\tilde{T}^{i}\right)^{-1}\right] .
$$

Thus

$$
\gamma\left(T^{n}\right)^{-1} \leq(1+\varepsilon) \gamma\left(\tilde{T}^{n}\right)^{-1}+\sum_{i=0}^{n-1}(1+\varepsilon)^{2} \gamma\left(T_{1}^{n-i}\right)^{-1}\left[\|T\| \gamma\left(\tilde{T}^{i+1}\right)^{-1}+\gamma\left(\tilde{T}^{i}\right)^{-1}\right] .
$$

Find $n_{0}$ such that

$$
\gamma\left(T_{1}^{i}\right) \geq(s-\varepsilon)^{i}, \quad \gamma\left(\tilde{T}^{i}\right) \geq(s-\varepsilon)^{i} \quad\left(i \geq n_{0}\right) .
$$

Denote

$$
K=\max _{1 \leq i \leq n_{0}+1} \max \left\{\gamma\left(T_{1}^{i}\right)^{-1}, \gamma\left(\tilde{T}^{i}\right)^{-1},(s-\varepsilon)^{-i}\right\} .
$$

For $n$ large enough we have

$$
\begin{aligned}
& \gamma\left(T^{n}\right)^{-1} \leq(1+\varepsilon)^{2}\left[(s-\varepsilon)^{-n}+\sum_{i=n_{0}}^{n-n_{0}-1}(s-\varepsilon)^{i-n}\left(\|T\|(s-\varepsilon)^{-i-1}+(s-\varepsilon)^{-i}\right)\right. \\
& \left.+\sum_{i=0}^{n_{0}-1}(s-\varepsilon)^{i-n}(\|T\| \cdot K+K)+\sum_{i=n-n_{0}}^{n-1} K\left(\|T\|(s-\varepsilon)^{-i-1}+(s-\varepsilon)^{-i}\right)\right] \\
& \leq(1+\varepsilon)^{2}(s-\varepsilon)^{n_{0}-n}\left[K+\left(n-2 n_{0}\right)(K \cdot\|T\|+K)+2 n_{0} K(\|T\| \cdot K+K)\right] \\
& \leq(1+\varepsilon)^{2}(s-\varepsilon)^{n_{0}-n} n \cdot K^{\prime}
\end{aligned}
$$

where $K^{\prime}$ is a constant independent of $n$. Hence

$$
\liminf _{n \rightarrow \infty} \gamma\left(T^{n}\right)^{1 / n} \geq \liminf _{n \rightarrow \infty}(s-\varepsilon)^{\frac{n-n_{0}}{n}}=s-\varepsilon .
$$

Since $\varepsilon>0$ was arbitrary, we conclude that $\liminf _{n \rightarrow \infty} \gamma\left(T^{n}\right)^{1 / n} \geq s$, so that

$$
\lim _{n \rightarrow \infty} \gamma\left(T^{n}\right)^{1 / n}=s
$$


Theorem 3. Let $T \in B(X)$ be s-regular. Then

$$
\operatorname{dist}\left\{0, \sigma_{\gamma}(T)\right\}=\lim _{n \rightarrow \infty} \gamma\left(T^{n}\right)^{1 / n} .
$$

Proof. Denote $r=\operatorname{dist}\left\{0, \sigma_{\gamma}(T)\right\}$. Let $M=R^{\infty}(T), T_{1}=T \mid M$, and let $\tilde{T}$ : $X / M \rightarrow X / M$ be the operator induced by $T$. If $\lambda$ is a complex number satisfying

$$
|\lambda|<\lim _{n \rightarrow \infty} \gamma\left(T^{n}\right)^{1 / n}=\min \left\{\lim _{n \rightarrow \infty} \gamma\left(T_{1}^{n}\right)^{1 / n}, \lim _{n \rightarrow \infty} \gamma\left(\tilde{T}^{n}\right)^{1 / n}\right\},
$$

then $T_{1}-\lambda$ is onto and $\tilde{T}-\lambda$ is bounded below. Thus $T-\lambda$ is s-regular by Lemma 1 and $\lim _{n \rightarrow \infty} \gamma\left(T^{n}\right)^{1 / n} \leq r$.

Conversely, it is well known (see e.g. [15, Theorem 5.2]) that $R^{\infty}(T-\lambda)$ is constant on the component of $\mathbf{C} \backslash \sigma_{\gamma}(T)$ containing 0, in particular $R^{\infty}(T-\lambda)=M$ for $|\lambda|<r$. If $|\lambda|<r$, then $(T-\lambda) M=M$ and $\tilde{T}-\lambda=\widetilde{T-\lambda}: X / M \rightarrow X / M$ is bounded below. Thus $\lim _{n \rightarrow \infty} \gamma\left(T_{1}^{n}\right)^{1 / n} \geq r$ and $\lim _{n \rightarrow \infty} \gamma\left(\tilde{T}^{n}\right)^{1 / n} \geq r$. Hence $\lim _{n \rightarrow \infty} \gamma\left(T^{n}\right)^{1 / n} \geq r$ by Lemma 2 .

Remark. It is possible to deduce the inequality dist $\left\{0, \sigma_{\gamma}(T)\right\} \geq \lim _{n \rightarrow \infty} \gamma\left(T^{n}\right)^{1 / n}$ from [11, Theorem 2.10]. We have obtained a new direct proof of this result.

Definition. $T \in B(X)$ is called essentially s-regular if $R(T)$ is closed and there exists a finite-dimensional subspace $F \subset X$ such that $N(T) \subset R^{\infty}(T)+F$.

Define further $\sigma_{e \gamma}(T)=\{\lambda \in \mathbf{C}: T-\lambda$ is not essentially s-regular $\}$.

For properties of essentially s-regular operators and the set $\sigma_{e \gamma}(T)$ see $[14,15]$.

Theorem 4. Let $T \in B(X)$ be essentially s-regular. Then $\lim _{n \rightarrow \infty} \gamma\left(T^{n}\right)^{1 / n}$ exists and

$$
\begin{aligned}
\lim _{n \rightarrow \infty} \gamma\left(T^{n}\right)^{1 / n} & =\max \{r: T-\lambda \text { is s-regular for } 0<|\lambda|<r\} \\
& =\operatorname{dist}\left\{0, \sigma_{\gamma}(T) \backslash\{0\}\right\} .
\end{aligned}
$$

Proof. By [14, Theorem 3.1] or [15, Theorem 2.1] there exist subspaces $X_{1}, X_{2} \subset X$ such that $X=X_{1} \oplus X_{2}, \operatorname{dim} X_{1}<\infty, T X_{1} \subset X_{1}, T X_{2} \subset X_{2}, T_{1}=T \mid X_{1}$ is nilpotent and $T_{2}=T \mid X_{2}$ is s-regular (the Kato decomposition). By the previous theorem $\operatorname{dist}\left\{0, \sigma_{\gamma}\left(T_{2}\right)\right\}=\lim _{n \rightarrow \infty} \gamma\left(T_{2}^{n}\right)^{1 / n}$. For $n \geq \operatorname{dim} X_{1}$ we have $T_{1}^{n}=0$ so that $N\left(T^{n}\right)=X_{1} \oplus N\left(T_{2}^{n}\right)$. Let $P$ be the projection with $R(P)=X_{2}$ and $N(P)=X_{1}$. Let $x_{2} \in X_{2}$. We have

$$
\begin{aligned}
\operatorname{dist}\left\{x_{2}, N\left(T_{2}^{n}\right)\right\} & =\inf \left\{\left\|x_{2}-y_{2}\right\|: y_{2} \in X_{2}, T_{2}^{n} y_{2}=0\right\} \\
& \leq\|P\| \inf \left\{\left\|y_{1} \oplus\left(x_{2}-y_{2}\right)\right\|: y_{1} \in X_{1}, y_{2} \in X_{2}, T_{2}^{n} y_{2}=0\right\} \\
& =\|P\| \operatorname{dist}\left\{x_{2}, N\left(T^{n}\right)\right\} \leq\|P\| \operatorname{dist}\left\{x_{2}, N\left(T_{2}^{n}\right)\right\} .
\end{aligned}
$$

Then

$$
\begin{aligned}
\gamma\left(T_{2}^{n}\right) & =\inf \left\{\frac{\left\|T_{2}^{n} x_{2}\right\|}{\operatorname{dist}\left\{x_{2}, N\left(T_{2}^{n}\right)\right\}}: x_{2} \in X_{2} \backslash N\left(T_{2}^{n}\right)\right\} \\
& \leq \inf \left\{\frac{\left\|T^{n} x_{2}\right\|}{\operatorname{dist}\left\{x_{2}, N\left(T^{n}\right)\right\}}: x_{2} \in X_{2} \backslash N\left(T^{n}\right)\right\} \\
& =\inf \left\{\frac{\left\|T^{n}\left(x_{1} \oplus x_{2}\right)\right\|}{\operatorname{dist}\left\{x_{1} \oplus x_{2}, N\left(T^{n}\right)\right\}}: x_{1} \oplus x_{2} \in X \backslash N\left(T^{n}\right)\right\}=\gamma\left(T^{n}\right)
\end{aligned}
$$


and

$$
\begin{aligned}
\gamma\left(T^{n}\right) & \leq \inf \left\{\frac{\left\|T_{2}^{n} x_{2}\right\|}{\operatorname{dist}\left\{x_{2}, N\left(T^{n}\right)\right\}}: x_{2} \in X_{2} \backslash N\left(T_{2}^{n}\right)\right\} \\
& \leq\|P\| \inf \left\{\frac{\left\|T_{2}^{n} x_{2}\right\|}{\operatorname{dist}\left\{x_{2}, N\left(T_{2}^{n}\right)\right\}}: x_{2} \in X_{2} \backslash N\left(T_{2}^{n}\right)\right\}=\|P\| \gamma\left(T_{2}^{n}\right) .
\end{aligned}
$$

Hence $\lim _{n \rightarrow \infty} \gamma\left(T^{n}\right)^{1 / n}=\lim _{n \rightarrow \infty} \gamma\left(T_{2}^{n}\right)^{1 / n}$.

If $\lambda \neq 0$, then $T-\lambda$ is s-regular if and only if $T_{2}-\lambda$ is s-regular. Then

$\max \{r: T-\lambda$ is s-regular for $0<|\lambda|<r\}=\operatorname{dist}\left\{0, \sigma_{\gamma}\left(T_{2}\right)\right\}=\lim _{n \rightarrow \infty} \gamma\left(T^{n}\right)^{1 / n}$

The following lemma is an analog of Lemma 1 for essentially s-regular operators:

Lemma 5. $T \in B(X)$ is essentially s-regular if and only if there exists a closed subspace $M \subset X$ such that $T M=M$ and the operator $\tilde{T}: X / M \rightarrow X / M$ induced by $T$ is upper semi-Fredholm.

Proof. If $T$ is essentially s-regular, then set $M=R^{\infty}(T)$. If $X=X_{1} \oplus X_{2}$ is the Kato decomposition $\left(\operatorname{dim} X_{1}<\infty, T X_{1} \subset X_{1}, T X_{2} \subset X_{2}, T_{1}=T \mid X\right.$ nilpotent and $T_{2}=T \mid X_{2}$ s-regular), then $M=R^{\infty}\left(T_{2}\right) \subset X_{2}$ and $T M=T_{2} M=M$. If $x=x_{1} \oplus x_{2}$ satisfies $T x \in M$, then $T_{2} x_{2} \in M$ so that $x_{2} \in M$. Thus $x \in X_{1}+M$ and $N(\tilde{T}) \subset X_{1}+M$. Hence $\operatorname{dim} N(\tilde{T})<\infty$.

Let $\pi: X \rightarrow X / M$ be the canonical projection. Since $M \subset R(T)$ and $R(\tilde{T})=$ $\{T x+M: x \in X\}=\pi R(T)$, the range of $\tilde{T}$ is closed. Thus $\tilde{T}$ is upper semiFredholm.

Conversely, let $M$ be a subspace of $X$ with the required properties. We can prove that $R(T)$ is closed in exactly the same way as in Lemma 1.

Further, $M \subset R^{\infty}(T)$. If $T x=0$, then $\tilde{T}(x+M)=0$, i.e. $\pi x \in N(\tilde{T})$. Thus $N(T) \subset \pi^{-1} N(\tilde{T}) \subset M+F \subset R^{\infty}(T)+F$ for a finite-dimensional subspace $F \subset X$.

Theorem 6. Let $T, A \in B(X), T A=A T$, and let $A$ be a quasinilpotent. Then

(1) $\sigma_{\gamma}(T+A)=\sigma_{\gamma}(T)$

(2) $\sigma_{\gamma e}(T+A)=\sigma_{\gamma e}(T)$.

Proof. Let $T$ be an essentially s-regular operator, and let $A$ be a quasinilpotent commuting with $T$. Denote $M=R^{\infty}(T), T_{1}=T \mid M$, and let $\tilde{T}: X / M \rightarrow X / M$ be the operator induced by $T$. Clearly $A M \subset M$ so that we can define operators $A_{1}=$ $A \mid M$ and $\tilde{A}: X / M \rightarrow X / M$ induced by $A$. Clearly $r\left(A_{1}\right)=\lim _{n \rightarrow \infty}\left\|A_{1}^{n}\right\|^{1 / n} \leq$ $\lim _{n \rightarrow \infty}\left\|A^{n}\right\|^{1 / n}=0$ and $r(\tilde{A})=\lim _{n \rightarrow \infty}\left\|\tilde{A}^{n}\right\|^{1 / n} \leq \lim _{n \rightarrow \infty}\left\|A^{n}\right\|^{1 / n}=0$ so that $\sigma\left(A_{1}\right)=\{0\}$ and $\sigma(\tilde{A})=\{0\}$. Further $T_{1} A_{1}=A_{1} T_{1}$ and $\tilde{T} \tilde{A}=\tilde{A} \tilde{T}$. Denote by

$$
\begin{aligned}
\sigma_{\delta}(T) & =\{\lambda \in \mathbf{C}: T-\lambda \text { is not onto }\}, \\
\sigma_{\pi}(T) & =\{\lambda \in \mathbf{C}: T-\lambda \text { is not bounded below }\}, \\
\sigma_{\pi e}(T) & =\{\lambda \in \mathbf{C}: T-\lambda \text { is not upper semi-Fredholm }\}
\end{aligned}
$$

the defect spectrum, the approximate point spectrum and the essential approximate point spectrum, respectively. 
By the spectral mapping property for these spectra we have

$$
\begin{aligned}
\sigma_{\delta}\left(T_{1}+A_{1}\right) & =\sigma_{\delta}(T), \\
\sigma_{\pi}(\tilde{T}+\tilde{A}) & =\sigma_{\pi}(\tilde{T}), \\
\sigma_{\pi e}(\tilde{T}+\tilde{A}) & =\sigma_{\pi e}(\tilde{T}) .
\end{aligned}
$$

Thus $0 \notin \sigma_{\delta}(T+A)$, i.e. $(T+A) M=M$. Similarly $0 \notin \sigma_{\pi e}(\tilde{T}+\tilde{A})$, i.e. $\tilde{T}+\tilde{A}$ is upper semi-Fredholm. By the previous lemma $T+A$ is essentially s-regular. This proves (2).

If $T$ is s-regular and $A$ a quasinilpotent commuting with $T$, then in the same way $(T+A) M=M$ and $\tilde{T}+\tilde{A}$ is bounded below. Hence $T+A$ is s-regular by Lemma 1.

Remark. Statement (1) for Hilbert space operators was proved in [10, Theorem 4.8]. The second statement gives a positive answer to Question 3 of [15].

\section{REFERENCES}

1. C. Apostol, The reduced minimum modulus, Michican Math. J. 32 (1985), 279-294. MR 87a:47003

2. K.-H. Förster and M.A. Kaashoek, The asymptotic behaviour of the reduced minimum modulus of a Fredholm operator, Proc. Amer. Math. Soc. 49 (1975), 123-131. MR 51:8867

3. M.A. Goldman and S.N. Kratchovski, On the stability of some properties of a class of linear operators, Soviet Math. Dokl. 14 (1973), 502-592; Dokl. Akad. Nauk SSSR 209 (1973), 769-772. MR 48:910

4. S. Grabiner, Uniform ascent and descent of bounded operators, J. Math. Soc. Japan 34 (1982), 317-337. MR 84a:47003

5. M.A. Kaashoek, Stability theorems for closed linear operators, Indag. Math. 27 (1965), 452466. MR 31:6129

6. T. Kato, Perturbation theory for nullity, deficiency and other quantities of linear operators, J. Anal. Math. 7 (1958), 261-322. MR 21:6541

7. _ Perturbation theory for linear operators, Springer-Verlag, Berlin, 1966. MR 34:3324

8. J.P. Labrouse, Les opérateurs quasi-Fredholm: une généralisation des opérateurs semiFredholm, Rend. Circ. Math. Palermo 29 (1989), 69-105.

9. E. Makai Jr. and J. Zemánek, The surjectivity radius, packing numbers and boundedness below of linear operators, Int. Eq. Oper. Th. 6 (1983), 372-384. MR 84m:47005

10. M. Mbekhta, Résolvant généralisé et théorie spectrale, J. Oper. Theory 21 (1989), 69-105. MR 91a:47004

11. M. Mbekhta and A. Ouahab, Opérateur s-régulier dans un espace de Banach et théorie spectrale, vol. 22, No. XII, Pub. Irma, Lille, 1990.

12. _ Contribution à la théorie spectrale généralisée dans les espaces de Banach, C. R. Acad. Sci. Paris 313 (1991), 833-836. MR 92h:47005

13. V. Müller, The inverse spectral radius formula and removability of spectrum, Cas. Pest. Mat. 108 (1983), 412-415. MR 85f: 46090

14.

15. V. Rakoćevič, Generalized spectrum and commuting compact perturbations, Proc. Edinb. Math. Soc. 36 (1993), 197-209. MR 94g:47012

16. P. Saphar, Contributions à l'étude des aplications linéaires dans un espace de Banach, Bull. Soc. Math. France 92 (1964), 363-384. MR 32:4549

17. J. Zemánek, The stability radius of a semi-Fredholm operator, Int. Eq. Oper. Th. 8 (1985), 137-144. MR 86c:47014

Institute of Mathematics AV ČR, Žitná 25, 11567 Praha 1, Czech Republic

E-mail address: vmuller@mbox.cesnet.cz 